\title{
Estimating Reliability Bounds on Industrial Plants
}

\author{
Andreas M. Hein \\ Laboratoire Genie Industriel, CentraleSupélec, \\ Université Paris-Saclay ${ }^{1}$ \\ Châtenay-Malabry, Paris region, France / \\ Paris-Saclay Energy Efficiency (PS2E) \\ Loges en Josas, Paris region, France
}

\author{
Romain Farel \\ Paris-Saclay Energy Efficiency (PS2E) \\ Loges en Josas, Paris region, France
}

\begin{abstract}
Reliability analysis is particularly relevant for industrial plants where plant failures can lead to large financial losses. Existing reliability analysis approaches mostly rely on heavyweight simulations that are computationally expensive and require extensive modeling effort. On the other hand, there is an industrial need for quickly evaluating plant reliability for developing new services and business models. In this paper, we extend and apply the reliability bound approach using linear programming to address this need. The reliability bound approach is based on a system model in the form of a graph, an event vector, and estimates for component reliabilities. Based on this model, lower and upper reliability bounds are calculated by solving a linear programming problem. The advantage of this approach is the ubiquity of solvers for linear programming. Furthermore, the approach is guaranteed to produce the narrowest bound with respect to the reliability data. We demonstrate the applicability of the approach to a subsystem of an industrial plant as a test case. Future work consists applying the method to whole plants and comparing the results with simulation-based approaches. Moreover, the approach is planned to be extended to system attributes such as buffers and multiple failure states.
\end{abstract}

\section{INTRODUCTION}

The reliability of industrial plants is a crucial for businesses to offer services and products. It is particularly important in the utilities business where the reliability of supplying a product has a significant impact on the business model. The same product supplied with different degrees of reliability can be treated as different products. Furthermore, being able to offer gas or electricity with a guaranteed reliability can be a decisive competitive advantage. Despite the importance of reliability in the utilities business, reliability analysis in the early stages of development has been mostly limited to qualitative methods such as the failure mode and effects analysis [1]. Few publications have considered quantitative reliability assessment for the early stages of product or service development such as [2], [3] for the automotive industry. [4] proposed a quantitative reliability analysis method for the early stage development of cyber-physical systems.

An overview of reliability analysis approaches for industrial plants is presented in [5], [6]. The presented approaches are not well-suited for addressing the goal of assisting reliability analysis for business model development. More specifically, there are two challenges: First, at an early stage of business model development, often detailed reliability data is not available or the reliability data is heterogeneous, i.e. for some components detailed data is available but for other, less mature components, only estimates are available. Second, most simulation-based methods require customized tools and expertise for using the tools but also for interpreting the results.

In this paper, we address these challenges by applying the reliability bound approach introduced by [7] to the plant reliability problem. Existing publications using the approach apply it to rather academic examples. To the authors' knowledge, there are no publications that demonstrate the applicability of the approach to industrial problems. In this paper, we demonstrate that the approach can be applied to high-level reliability analysis of industrial plants. We extend the method by automatizing parts of the otherwise tedious formulation of the reliability problem. Finally, we propose avenues for future research for solving more advanced reliability analysis problems with this approach.

\section{BACKGROUND: RELIABILITY BOUND APPROACH}

The reliability bound approach has been introduced by Song et al. in [7]. The main advantage of the reliability bound approach is that it is guaranteed to find the upper and lower bounds of system reliability, if a solution exists, as it is based on linear programming. In addition, solvers for linear programming problems are ubiquitous for even very large problems. Hence, the problem can be solved with most of the commercially and freely available linear programming solvers. This is in contrast to simulation-based approaches (Monte Carlo simulation [8], Bayesian networks, Markov Chains [9]) that usually cannot guarantee to find the solution. Furthermore, the component and subsystem data used as the input can be of various degrees of accuracy. Rough reliability estimates can be combined with probability distributions from extensive statistical data. A significant drawback is that the number of variables for describing the problem increases exponentially with the number of components in the system.

In [7] the reliability boundary approach was applied to several academic case studies such as a truss structure, a beam suspended by perfectly fragile wires, and a rigid plastic structure.

${ }^{1}$ Formerly Laboratoire Genie Industriel, Ecole Centrale Paris 
These case studies are intended to showcase the applicability of the method to various reliability engineering problems in structural mechanics. [10] extends the approach to more complex systems that can be represented a multiple levels by decomposing a system into subsystems and then aggregating individual subsystem reliabilities into a system-level reliability value. [11] use the approach for analyzing the effect of an earthquake to a lifeline network.

In reliability analysis, we are interested in the reliability of a system with $n$ components. We denote the state of the system as $E_{\text {sys. }}$. The system state can be the failure state or the state of nominal operation. We introduce a vector $\boldsymbol{E}_{i}=\left(E_{i 1}, E_{i 2}, \ldots\right)$ with vectors $E_{i m}$ where $i$ denotes a component and $m$ the mth state of the component. We assert that a system state depends on the states of the components:

$$
E_{\text {system }}=L\left(\boldsymbol{E}_{\mathbf{1}}, \boldsymbol{E}_{2}, \ldots \boldsymbol{E}_{\boldsymbol{n}}\right)
$$

$L$ is a logical function which consists of "unions and intersections of component events or their complements." [7] We are interested in the probability of the system state $E_{\text {sys }}$, denoted as $P\left(E_{\text {system }}\right)$. For a system with parallel and serial elements, $P\left(E_{\text {system }}\right)$ can be calculated via the union and intersections of the component states:

$$
P\left(E_{\text {system }}\right)=P\left(\bigcup_{k} \bigcap_{i \in C_{k}} E_{i}\right)
$$

$C_{k}$ denotes the component state indices that constitute the kth cut set. Evaluating this formula for systems with a large number of components and states is computationally very expensive. Hence, there has been a persistent interest in developing formulas for upper and lower reliability bounds. A reliability bound can be formally defined as:

$$
P_{\min } \leq P\left(E_{\text {system }}\right) \leq P_{\max }
$$

$P_{\min }$ is the minimum probability bound and $P_{\max }$ the maximum probability bound. [7] shows that linear programming can be used for not only finding the reliability bounds for a general system but also to guarantee finding the narrowest possible probability bounds. The linear programming problem can be formulated as:

$$
\begin{aligned}
& \text { Minimize / maximize } \boldsymbol{c}^{T} \boldsymbol{p} \\
& \text { Subject to the equality constraints } \boldsymbol{a}_{1} \boldsymbol{p}=\boldsymbol{b}_{1} \\
& \text { And the inequality constraints } \boldsymbol{a}_{2} \boldsymbol{p} \geq \boldsymbol{b}_{2}
\end{aligned}
$$

$\boldsymbol{p}$ is a vector of variables and $\boldsymbol{c}^{T}$ the transpose of a vector of coefficients. The coefficients correspond to the Boolean function of the system. For example, for a serial system with two components, this would result in:

$$
\begin{gathered}
\boldsymbol{p}=\left[P\left(E_{1} E_{2}\right), P\left(\overline{E_{1}} E_{2}\right), P\left(E_{1} \overline{E_{2}}\right) P\left(\left(\overline{E_{1} E_{2}}\right)\right)\right] \\
\boldsymbol{c}^{T}=[0,1,1,1]
\end{gathered}
$$

Where 0 in the vector $\boldsymbol{c}^{T}$ indicates that the system is operating nominally and 1 indicates that the system has failed. The event $E_{i}$ for component $i$ indicates a nominally operating component. The complement $\bar{E}_{i}$ indicates a component failure event. For a serial system this means that only when both components work, then the system works.

$\boldsymbol{a}$ and $\boldsymbol{b}$ are coefficient vectors and matrices. The solution of the linear programming problem is a feasible vector $p$ that minimizes (maximizes) the objective function $\boldsymbol{c}^{T} \boldsymbol{p}$.

Fig. 1 depicts the steps in the reliability analysis method. First, the equality constraint coefficient matrix is constructed, using the power set of component states. In the second step, the linear programming problem is solved.

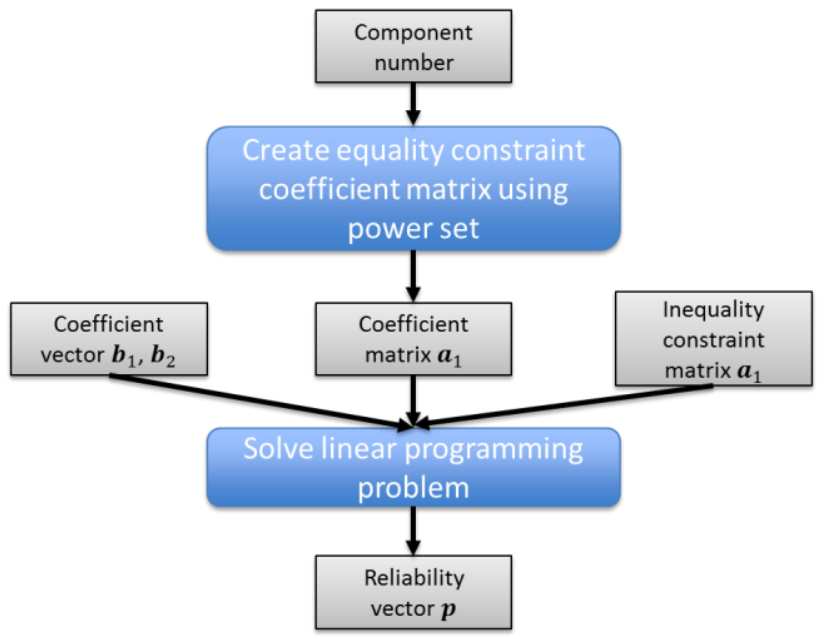

Fig. 1: Visualization of the reliability analysis steps

\section{AUTOMATED SYSTEM MODEL GENERATION}

The existing reliability bound approach is practically difficult to use, as it provides the mathematical basis for solving the formulating the linear programming problem. However, as the size of the event space increases exponentially, manually constructing the coefficient matrices becomes increasingly difficult. In order to solve this problem, we extend the original linear programming problem by automatizing the generation of the problem formulation.

The main challenge is the exponential growth of the cardinality of the event set (the number of events in the set) by $2^{n}$. For a small system with 10 components, this leads to 1024 events. Hence, the size of the equality constraints matrix is 1024 $\mathrm{x}$ 1024. We develop an algorithm for automatically creating the equality constraints matrix, which increases proportionally with the cardinality of the events set.

We illustrate the algorithm for a 2-component, serial system. The event space in the form of a Venn diagram is shown in Fig. 2. $E_{1}$ is the event that component 1 fails. $E_{2}$ is the event that component 2 fails. $\overline{E_{1} \cup E_{2}}$ is the event that both 
components function. We subdivide the areas in the Venn diagram into four partitions $p_{1}$ to $p_{4}$. The event that the system fails is the union of the partitions $p_{1}, p_{2}, p_{3}$ or alternatively the union $E_{1} \cup E_{2}$. Why is this relevant? It is relevant as in most cases we only have reliability data for the components $E_{1}, E_{2}$, and we need to calculate the probability $\left(E_{1} \cap E_{2}\right)$ of both components failing and each of the components failing separately $\left(E_{1} \cap \overline{E_{2}}\right.$ and $\left.\overline{E_{1}} \cap E_{2}\right)$. We therefore need to form the union of all these sets that gives us the failure event set for the system.

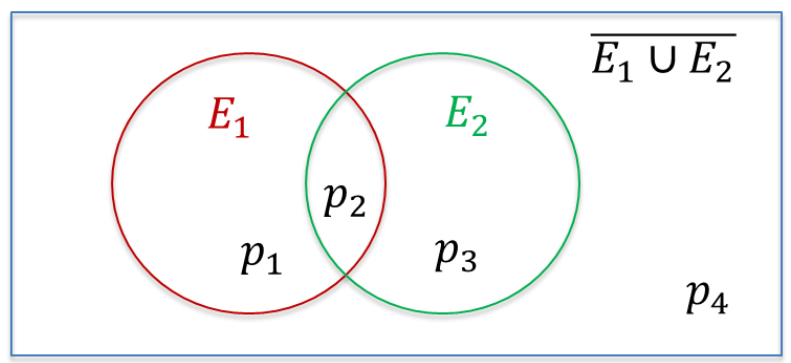

Fig. 2: Venn diagram for a 2-element serial system

Table 1 shows the equality constraints matrix for the 2 element serial system.

Table 1: Equality constraint matrix $\boldsymbol{a}_{1}$

\begin{tabular}{|c|c|c|c|c|}
\hline & $p_{1}$ & $p_{2}$ & $p_{3}$ & $p_{4}$ \\
\hline & $E_{1} \cap \overline{E_{2}}$ & $E_{1} \cap E_{2}$ & $\overline{E_{1}} \cap E_{2}$ & $\overline{E_{1} \cup E_{2}}$ \\
\hline$E_{1}$ & 1 & 1 & 0 & 0 \\
\hline$E_{2}$ & 0 & 1 & 1 & 0 \\
\hline
\end{tabular}

As a third row in the constraint matrix, the following equality constraint is added which is one of the Kolmogorov axioms of probability theory:

$$
\sum_{i=1}^{4} p_{i}=1
$$

We can map the set-theoretic expressions for $p_{i}$ in Table 1 by mapping each of the expressions to a set of its non-conjunctive elements.

$$
\begin{gathered}
\left\{E_{1}, \overline{E_{2}}\right\} \rightarrow\left\{E_{1}\right\} \\
\left\{E_{1}, E_{2}\right\} \rightarrow\left\{E_{1}, E_{2}\right\} \\
\left\{\overline{E_{1}}, E_{2}\right\} \rightarrow\left\{E_{2}\right\} \\
\left\{\overline{E_{1}}, \overline{E_{2}}\right\} \rightarrow\{\}
\end{gathered}
$$

This results in the set of all subsets, i.e. the power-set of the elements $\wp(\mathrm{S})$ with $S=\left\{E_{1}, E_{2}\right\}$. The power set is hence:

$$
\wp(S)=\left\{\{\},\left\{E_{1}\right\},\left\{E_{2}\right\},\left\{E_{1}, E_{2}\right\}\right\}
$$

The power set can be easily generated algorithmically via the binomial distribution. Using the power set, we can then formulate an algorithm for creating the constraint matrix for all single and multiple events by simply writing a " 1 " entry into the matrix when the event is present in a subset of the power set and a " 0 " when it is not. We can therefore, in principle, create the coefficient matrix for the equality constraints automatically, which is very useful for large coefficient matrices.

The coefficient vector $\boldsymbol{c}^{T}$ is created by using the Boolean function for the system For the 2-element serial system the corresponding Boolean function is:

$$
\overline{E_{1}} A N D \overline{E_{2}} \rightarrow E_{s y s}
$$

The Boolean function for combined serial and parallel systems can be easily generated by a combination of logical AND and OR expressions from Boolean algebra.

\section{APPLICATION TO INDUSTRIAL PLANT CASE STUDY}

We apply the reliability analysis method presented in the previous section to an existing air separation unit (ASU) in an industrial plant. The ASU consists of 5 serial components and two separate parallel components of 2 identical components as shown in Fig. 3. Annual failure probabilities are included. As the original values are confidential, random order of magnitude values are used.

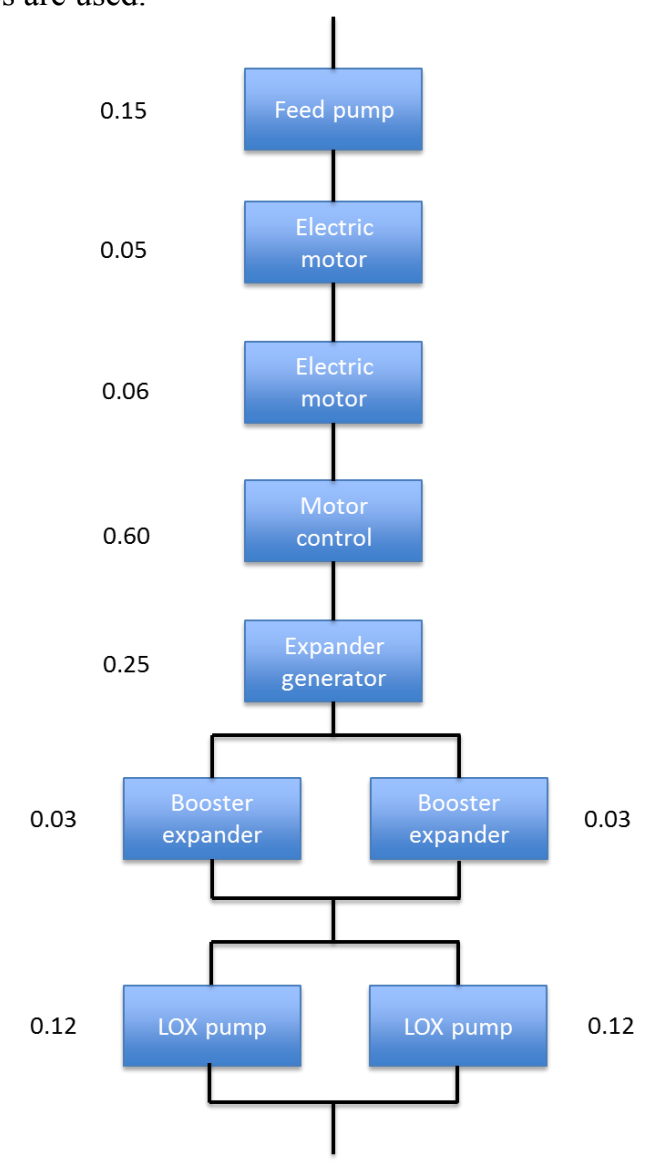

Fig. 3: Air Separation Unit component diagram with annual failure probabilities 
First, we enumerate the complete power set of the event space of 512 entries and create the coefficient matrix ( $512 \times 512$ entries) for the equality constraints. Component reliability data for all eight components is entered into the probability vector $\boldsymbol{b}_{1}$ :

$\boldsymbol{b}_{1}^{T}$

$=[0.15,0.05,0.06,0.60,0.25,0.03,0.03,0.12,0.12 \ldots, 0, \ldots, 1]$

The final entry " 1 " in the vector represents the equality constraint for the sum of all probabilities, including all complements, which is by definition 1 . Most of the entries in the vector are 0 , as only the component probabilities and the sum of probabilities are known. A solution can still be found if the basic conditions for finding a solution in linear programming are satisfied. This means that the solution space is a convex hypercube and certain pathological constraints do not occur.

\section{RESULTS}

Running the simulation yields the following lower and upper bounds for the system:

$$
E_{s y s_{-} \min } \leq E_{\text {sys }} \leq E_{\text {sys_max }}
$$

With

$$
\begin{aligned}
& E_{\text {sys_min }}=0.0282 \\
& E_{\text {sys_max }}=0.0649
\end{aligned}
$$

$$
E_{\text {sys_min }} \leq E_{\text {sys }} \leq E_{\text {sys_max }}
$$

\section{CONCLUSIONS}

We have demonstrated that the reliability bound approach can be applied to a practical industrial reliability case for a quick reliability estimate of an industrial plant. Future work will focus on extending the approach to model typical industrial plant attributes such as systems with buffers such as tanks and treating different failure modes such as short and long duration failures.

\section{REFERENCES}

[1] E. Lodgaard and Ø. Pellegård, "Failure Mode and Effects Analysis in Combination with the Problem Solving A3," in Proceedings of the 18th International Conference on Engineering Design (ICED 11), Impacting Society through Engineering Design, Vol. 9: Design Methods and Tools pt. 1, 2011.

[2] M. Kopp, D. Hofmann, and B. Bertsche, "Early Reliability Estimation In Automotive Industry," in $D S$ 68-10: Proceedings of the 18th International Conference on Engineering Design (ICED 11), Impacting Society through Engineering Design, Vol. 10: Design Methods and Tools pt. 2, 2011.
[3] M. Kopp, "Modularisierung und Synthese von Zuverlässigkeitsmethoden," Dissertation, University of Stuttgart, 2013.

[4] Y. Zhang and T. Kurtoglu, "System-Level Reliability Analysis for Conceptual Design of Electrical Power Systems," in Conference on Systems Engineering Research (CSER), 2011, pp. 15-16.

[5] J. Tixier, G. Dusserre, O. Salvi, and D. Gaston, "Review of 62 risk analysis methodologies of industrial plants," J. Loss Prev. Process Ind., vol. 15, no. 4, pp. 291-303, 2002.

[6] V. González, J. Gómez, and M. López, “Availability and reliability assessment of industrial complex systems: A practical view applied on a bioethanol plant simulation," in Safety, Reliability and Risk Analysis: Theory, Methods and Applications, S. Martorell, C. Guedes Soares, and J. Barnett, Eds. CRC Press, 2009, pp. 687-695.

[7] J. Song and A. Der Kiureghian, "Bounds on system reliability by linear programming," J. Eng. Mech., vol. 129, no. 6, pp. 627-636, 2003.

[8] M. Marseguerra and E. Zio, Basics of the Monte Carlo method with application to system reliability. LiLoLe Publishing, Hagen, 2002.

[9] H. Boudali and J. Dugan, "A discrete-time Bayesian network reliability modeling and analysis framework," Reliab. Eng. Syst. Saf., vol. 87, no. 3, pp. 337-349, 2005.

[10] A. Der Kiureghian and J. Song, "Multi-scale reliability analysis and updating of complex systems by use of linear programming," Reliab. Eng. Syst. Saf., vol. 93, no. 2, pp. 288-297, 2008.

[11] J. Song and S. Ok, "Multi-scale system reliability analysis of lifeline networks under earthquake hazards," Earthq. Eng. Struct. Dyn., vol. 39, no. 3, pp. 259-279, 2010. 